\title{
On the Prins reaction of terminal olefins and formaldehyde in trifluoroacetic acid
}

\author{
Hasanain A. A. Almohseni, Matthew A. H. Stent, David M. Hodgson,* \\ Department of Chemistry, University of Oxford, Chemistry Research Laboratory, Mansfield Road, Oxford, OX1 3TA, UK; e-mail: \\ david.hodgson@chem.ox.ac.uk
}

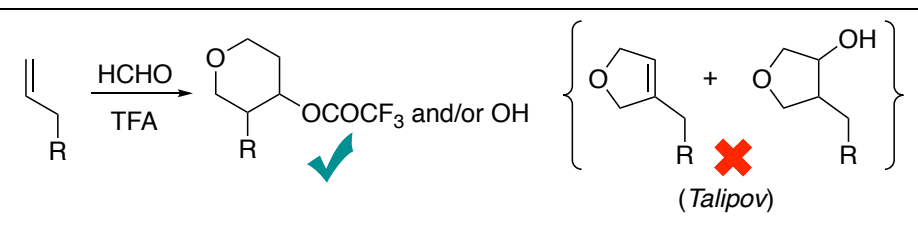

The Prins reaction of 1-heptene, as a representative terminal alkene, with formaldehyde in trifluoroacetic acid produces 3-butyl-4(trifluoroacetoxy)tetrahydropyran and/or 3-butyl-4-hydroxytetrahydropyran; it does not provide (as previously reported by Talipov and co-workers) a route to 3-alkyl-2,5-dihydrofurans and 4-alkyl-3-hydroxytetrahydrofurans.

Keywords: Prins reaction, terminal alkenes, formaldehyde, trifluoroacetic acid, tetrahydropyrans.

Acid-induced reaction between homoallylic alcohols 1 and aldehydes (Prins cyclisation) provides a straightforward entry to substituted tetrahydropyrans 2 (Scheme 1 ). ${ }^{1}$ The original Prins reaction, using simpler terminal olefins $\mathbf{3}$, can produce a range of products depending on the substrate and experimental conditions. ${ }^{2}$ In general, the latter is not viewed as a useful strategy to tetrahydropyrans, although 3-alkyl-4chlorotetrahydropyrans $4(\mathrm{X}=\mathrm{Cl})$ are accessible in good yields from terminal olefins using paraformaldehyde and gaseous $\mathrm{HCl}$ at low temperature (Scheme 2). ${ }^{3}$ Using $\mathrm{H}_{2} \mathrm{SO}_{4}$ with formaldehyde and acetic acid or under aqueous conditions is known to generate 4-acetoxy- or 4-hydroxy- 3substituted tetrahydropyrans, together with significant quantities of 4-substituted-1,3-dioxanes. ${ }^{4}$

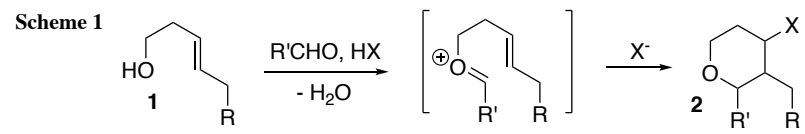

Scheme 2

Starting in in 1993, Talipov and co-workers claimed in a series of papers, ${ }^{5}$ a patent $^{6}$ and a review article, ${ }^{7}$ that switching to trifluoroacetic acid (TFA) as solvent with terminal olefins 3 and paraformaldehyde led, remarkably, to a mixture of 3-alkyl-2,5-dihydrofurans 5 and 4-alkyl-3hydroxytetrahydrofurans 6 (4:1 to 2:1, depending on water content) in good overall yields (Scheme 3 ). Further studies (including kinetic and computational) on this latter transformation were subsequently reported, even up to $2015 .^{8}$ This process was also reported as being successful for higher aldehydes (using $\mathrm{R}^{\prime} \mathrm{CHO}$ instead of $\mathrm{HCHO}$ ) giving 2,3,5-trisubstituted-2,5-dihydrofurans, ${ }^{5 \mathrm{~b}}$ and (in this Journal) as giving a mixture of 4-alkyl-3-trifluoroacetoxy- and 4alkyl-3-chloro-tetrahydrofurans with formaldehyde and $\mathrm{Me}_{3} \mathrm{SiCl}$ in TFA. ${ }^{9}$ Herein, we disclose our re-examination of the reaction of a terminal alkene with formaldehyde in TFA.<smiles>[R]CC1COCC1O</smiles>

The reaction of formaldehyde and 1-heptene (Scheme 3, $\mathrm{R}=$ butyl) in TFA was reported to give 3-pentyl-2,5dihydrofuran $5 \mathbf{a}(\mathrm{R}=\mathrm{Bu})$ in good yields and has been the most extensively investigated; ${ }^{5 a, b}$ consequently, this synthesis was re-examined. Following an exact experimental procedure of Talipov, which reportedly gave a $43 \%$ yield of 3-pentyl-2,5-dihydrofuran, ${ }^{5 b}$ 1-heptene was reacted with $\mathrm{HCHO}$ in TFA for $3 \mathrm{~h}$ at $20^{\circ} \mathrm{C}$. The major component we isolated was a non-polar colourless oil, possessing ${ }^{1} \mathrm{H} \mathrm{NMR}$ data [eg $\left(400 \mathrm{MHz}, \mathrm{CDCl}_{3}\right), \delta$, ppm: $\left.4.87(1 \mathrm{H}, \mathrm{td})\right]$ similar to the reported data [lit. ${ }^{5 \mathrm{a}, \mathrm{b}}:\left(80 \mathrm{MHz}, \mathrm{CDCl}_{3}\right), \delta$, ppm: $4.63-$ $5.09(1 \mathrm{H}, \mathrm{m}]$. However, the data is inconsistent with that for 3-pentyl-2,5-dihydrofuran (5a) [eg $^{10 \mathrm{a}}\left(60 \mathrm{MHz}, \mathrm{CCl}_{4}\right), \delta$, ppm: $5.4(1 \mathrm{H},=\mathrm{CH})]){ }^{10}$

Scheme 4

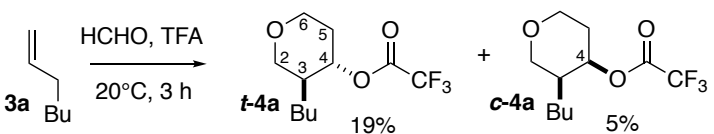

The material isolated under Prins conditions in anhydrous TFA is most straightforwardly assigned as tetrahydropyranyl trifluoroacetates $\boldsymbol{t} \mathbf{- 4 a}$ and $\boldsymbol{c - 4 a}$ (Scheme 4), which would arise from a reaction akin to Scheme $2\left(\mathrm{X}^{-}=\mathrm{F}_{3} \mathrm{CCO}_{2}^{-}\right)$. Indeed, our spectroscopic data is consistent with trifluoroacetate data reported by Talipov from the reaction mentioned above of terminal alkenes with formaldehyde and $\mathrm{Me}_{3} \mathrm{SiCl}$ in $\mathrm{TFA},{ }^{9}$ albeit Talipov assigned the products as 4alkyl-3-trifluoroacetoxytetrahydrofurans. Our 2D NMR data $\left({ }^{1} \mathrm{H}-{ }^{1} \mathrm{H}\right.$ COSY, DEPT-HSQC, and ${ }^{1} \mathrm{H}-{ }^{13} \mathrm{C} \mathrm{HMBC}$, see Supplementary information for spectra) provides strong evidence for the 3,4-disubstituted tetrahydropyran structure 
4a. For $\boldsymbol{t}-\mathbf{4 a},{ }^{1} \mathrm{H}-{ }^{1} \mathrm{H}$ COSY correlations between $\mathrm{H}-4$ and $\mathrm{H}-$ 3 and $\mathrm{H}_{\mathrm{a}}-5 / \mathrm{H}_{\mathrm{b}}-5$ revealed the existence of a $\mathrm{C}-3 / \mathrm{C}-4 / \mathrm{C}-5$ sequence, and the alkyl group is located at $\mathrm{C}-3$ based on DEPT-HSQC. ${ }^{1} \mathrm{H}-{ }^{1} \mathrm{H}$ COSY and DEPT-HSQC data showed that the protons at $\delta 3.19(1 \mathrm{H}$, dd) and one $\mathrm{H}$ of $\delta$ 3.93-4.01 $(2 \mathrm{H}, \mathrm{m})$ are the $\mathrm{C}-2$ methylene, whereas the protons at $3.50(1 \mathrm{H}, \mathrm{dd})$ and the second $\mathrm{H}$ of $\delta 3.93-4.01$ $(2 \mathrm{H}, \mathrm{m})$ are the $\mathrm{C}-6$ methylene. Both $\mathrm{H}-2 / \mathrm{C}-2$ and $\mathrm{H}-6 / \mathrm{C}-$ 6 are connected by an ether linkage based on $\mathrm{HMBC}$, and long-range HMBC correlations were observed between $\mathrm{H}-$ 4 and $\mathrm{C}-2$ and $\mathrm{C}-6$. That the major component $\boldsymbol{t}-\mathbf{4 a}$ possessed the trans configuration was based on the vicinal $J$ values for $\mathrm{H}-4$ being larger for $\boldsymbol{t}-\mathbf{4 a}(\mathrm{td}, J=9.5, J=4.5)$ compared with those observed for the minor cis-3,4disubstituted tetrahydropyran $\boldsymbol{c}-\mathbf{4 a}(\mathrm{q}, J=3.5)$.

Following correspondence with Talipov in 2008, he repeated the reaction between formaldehyde and 1-heptene in TFA, followed by neutralisation with ammonia (the latter would expected to cleave trifluoroacetates to alcohols). This gave a mixture of two compounds for which he kindly provided the ${ }^{13} \mathrm{C}$ NMR data, assigning them as cis- and trans-4-pentyl-3-hydroxytetrahydrofuran. We obtained essentially identical data following addition of ammonia at the end of the Prins reaction. The same data were obtained if the individual tetrahydropyranyl trifluoroacetates $\boldsymbol{t}-\mathbf{4 a}$ and $\boldsymbol{c}$ 4a underwent methanolysis in the presence of $\mathrm{K}_{2} \mathrm{CO}_{3}$, to give $\boldsymbol{t} \mathbf{- 4 b}$ and $\boldsymbol{c}-\mathbf{4 b}$. A comparison of this data with literature ${ }^{13} \mathrm{C}$ NMR data for 4-hydroxytetrahydropyrans ${ }^{11,12}$ and 3hydroxytetrahydrofurans ${ }^{12,13}$ is given in Fig. 1.

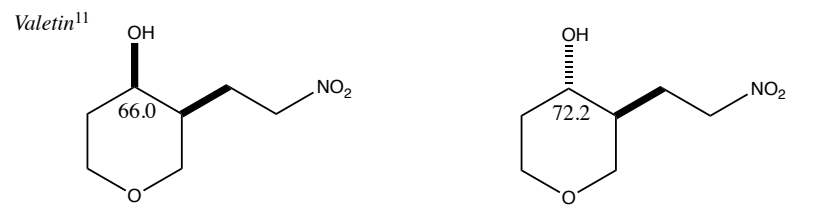

Current Work
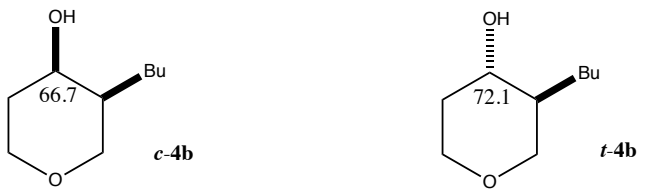

Parsons $^{12}$
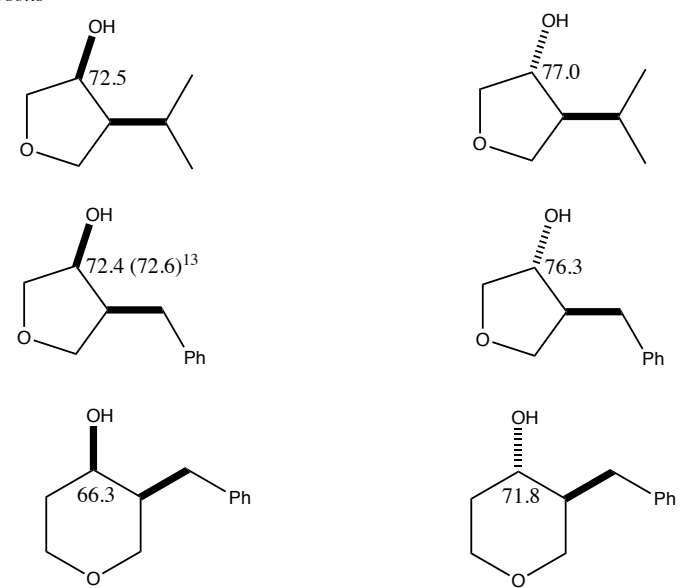

Figure 1. Comparison of selected ${ }^{13} \mathrm{C}$ NMR data for 3hydroxytetrahydrofurans and 4-hydroxytetrahydropyrans.

On analysing the ${ }^{13} \mathrm{C}$ NMR data in Fig. 1, it can been seen, in particular, that the carbinol carbons are always greater than 70 ppm for 4-alkyl-3-hydroxytetrahydrofurans, with the trans greater than $76 \mathrm{ppm}$. In contrast, the carbinol carbons of 3-alkyl-4-hydroxytetrahydropyrans are $\sim 66 \mathrm{ppm}$ and $\sim 72$ ppm for cis- and trans-isomers, respectively. This data comparison provides strong evidence that cis- and trans-4butyl-3-hydroxytetrahydropyran were prepared and not the 3-hydroxytetrahydrofurans, and further implies that the process proceeds as in Scheme 2 not Scheme 3 and by analogy this pathway is likely for higher aldehydes in TFA as well.

Finally, the data for 3-alkyl-4-chloro-tetrahydro'furans', obtained by Talipov as the additional product from terminal olefins with formaldehyde and $\mathrm{Me}_{3} \mathrm{SiCl}$ in $\mathrm{TFA},{ }^{9}$ was compared with data for a representative literature 3-alkyl-4chlorotetrahydropyran ${ }^{15}$ (Fig. 2). The data is consistant with the products from Talpov as also being the six- not five-ring ethers.

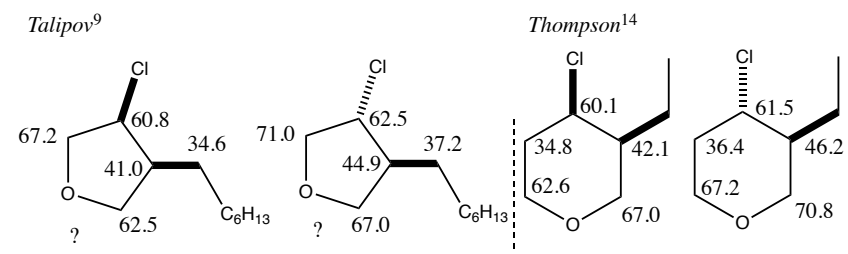

Figure 2. Comparison of selected ${ }^{13} \mathrm{C}$ NMR data for 3alkyl-4-chloro-tetrahydro'furans' and -tetrahydropyrans.

In summary, Prins reactions of terminal alkenes with formaldehyde in trifluoroacetic acid produce substituted tetrahydropyrans, and not the erroneously reported 2,5dihydrofurans or substituted tetrahydrofurans.

\section{Experimental}

Paraformaldehyde was dried overnight under high vacuum prior to use. 1-Heptene was freshly distilled from $\mathrm{CaH}_{2}$. Trifluoroacetic acid (99\%, extra pure) was used as received (Acros). Petrol (petroleum ether) of bp $40-60^{\circ} \mathrm{C}$ was used in flash column chromatography. Flash column chromatography was carried out using silica gel (VWR chemicals, BDH), monitored by thin layer chromatography (TLC) (Merck $60 \mathrm{~F}_{254}$ ) plates. TLC plates were viewed by immersion in $\mathrm{KMnO}_{4}$, followed by heating. Infrared spectra were obtained using a PerkinElmer FT-IR spectrometer (Universal ATR Sampling Accessory), with absorption maxima quoted in wavenumbers $\left(\mathrm{cm}^{-1}\right)$. Peak intensities are described as broad (br), weak (w), medium (m) or strong (s). Nuclear magnetic resonance $\left({ }^{1} \mathrm{H}\right.$ NMR, ${ }^{13} \mathrm{C}$ NMR, ${ }^{1} \mathrm{H}-{ }^{1} \mathrm{H}$ COSY, DEPT-HSQC, and ${ }^{1} \mathrm{H}-{ }^{13} \mathrm{C} \mathrm{HMBC}$ ) spectra were recorded on a Bruker Avance AVIIIHD 400 spectrometer in $\mathrm{CDCl}_{3}$, referenced to residual $\mathrm{CHCl}_{3}$ singlet at $\delta 7.26$, and to the central line of $\mathrm{CDCl}_{3}$ triplet at 77.16 for ${ }^{13} \mathrm{C} \mathrm{NMR}$ spectra. Proton coupling constants $(J)$ are measured to the nearest $0.5 \mathrm{~Hz}$. The splittings are quoted as singlet (s), doublet (d), triplet (t), quartet (q), doublet of doublets (dd), doublet of doublet of doublets (ddd), triplet of doublets (td), doublet of triplets (dt) or multiplet (m). ${ }^{13} \mathrm{C}$ NMR peaks were assigned by standard methods using HSQC. High resolution mass spectra were obtained by electrospray ionisation, using tetraoctylammonium bromide or sodium dodecyl sulfate as the lock mass. 
Trans- and cis-3-butyltetrahydro-2H-pyran-4-yl 2,2,2-trifluoroacetate $(\boldsymbol{t}-\mathbf{4 a}$ and $\boldsymbol{c}-\mathbf{4 a})$. The procedure and reaction scale of Talipov was followed. ${ }^{5 \mathrm{~b}}$ TFA $(15 \mathrm{ml})$ was added to paraformaldehyde $(5.00 \mathrm{~g}, 170 \mathrm{mmol})$, and the mixture heated until a clear solution formed (3-5 min). The mixture was cooled to $\mathrm{rt}$, then 1-heptene (3a) $(9.3 \mathrm{ml}, 80$ mmol) was added dropwise. After $3 \mathrm{~h}$ at $\mathrm{rt},{ }^{15}$ unreacted 1 heptene and TFA were removed by distillation $\left(<40^{\circ} \mathrm{C}\right.$, $\sim 100 \mathrm{mbar})$. The residue, a yellow oil $(\sim 11 \mathrm{~g})$, was distilled at $\sim 15 \mathrm{mbar}$ and the fraction of bp $60-70^{\circ} \mathrm{C}(5.3 \mathrm{~g}$, an impure 75:25 mixture of $\boldsymbol{t} \mathbf{- 4 a}$ and $\boldsymbol{c}-\mathbf{4 a})$ further purified by flash column chromatography $\left(0-20 \% \mathrm{Et}_{2} \mathrm{O}\right.$ in petrol). First eluted a colourless liquid, trans-3-butyltetrahydro- $2 H$-pyran-4-yl 2,2,2-trifluoroacetate $(\boldsymbol{t}-\mathbf{4 a})$. Yield $3.2 \mathrm{~g}(19 \%) . R_{\mathrm{f}}=0.26$ (20\% $\mathrm{Et}_{2} \mathrm{O}$ in petrol). IR spectrum (film), $\mathrm{v}, \mathrm{cm}^{-1}: 1249 \mathrm{~s}$, 1139 w, $1164 \mathrm{~s}, 1222 \mathrm{~m}, 1781 \mathrm{~s}, 2861 \mathrm{w}, 2934 \mathrm{~m}, 2962 \mathrm{~m}$. ${ }^{1} \mathrm{H}$ NMR spectrum $\left(400 \mathrm{MHz}, \mathrm{CDCl}_{3}\right), \delta, \operatorname{ppm}(J, \mathrm{~Hz}): 0.88$ $\left(3 \mathrm{H}, \mathrm{t}, J=7, \mathrm{CH}_{3}\right) ; 1.14-1.34\left(5 \mathrm{H}, \mathrm{m}, 8,9-\mathrm{CH}_{2}\right.$ and $\left.7-\mathrm{CH}_{\mathrm{a}}\right)$; 1.40-1.47 (1H, m, 7- $\left.\mathrm{CH}_{\mathrm{b}}\right)$; 1.71-1.79 (1H, m, 5- $\left.\mathrm{CH}_{\mathrm{a}}\right)$; 1.81-1.88 (1H, m, 3-CH); 2.02-2.08 (1H, m, 5-CHb); 3.19 $\left(1 \mathrm{H}, \mathrm{dd}, J=12, J=9,2-\mathrm{CH}_{\mathrm{a}}\right) ; 3.50(1 \mathrm{H}, \mathrm{ddd}, J=12, J=$ $\left.10.5, J=3,6-\mathrm{CH}_{\mathrm{a}}\right) ; 3.93-4.01\left(2 \mathrm{H}, \mathrm{m}, 2-\mathrm{CH}_{\mathrm{b}}\right.$ and $\left.6-\mathrm{CH}_{\mathrm{b}}\right)$; 4.87 (1H, td, $J=9.5, J=4.5,4-\mathrm{CH}) .{ }^{13} \mathrm{C}$ NMR spectrum $\left(100 \mathrm{MHz}, \mathrm{CDCl}_{3}\right), \delta$, ppm $(J, \mathrm{~Hz})$ : $13.9\left(\mathrm{CH}_{3}\right) ; 22.9(\mathrm{C}-9)$; 28.1 (C-7); 28.8 (C-8); 30.7 (C-5); 40.7 (C-3); 65.6 (C-6); $69.9(\mathrm{C}-2) ; 78.9(\mathrm{C}-4) ; 114.7\left(\mathrm{Q}, J_{\mathrm{C}-\mathrm{F}}=286, \mathrm{CF}_{3}\right), 157.3(\mathrm{q}$, $\left.J_{\mathrm{C}-\mathrm{F}}=42, \mathrm{C}=\mathrm{O}\right) .{ }^{19} \mathrm{~F}$ NMR spectrum $\left(376 \mathrm{MHz}, \mathrm{CDCl}_{3}\right), \delta$, ppm: -75.2. Found, $m / z: 277.1023[\mathrm{M}+\mathrm{Na}]^{+} . \mathrm{C}_{11} \mathrm{H}_{17} \mathrm{O}_{3} \mathrm{~F}_{3} \mathrm{Na}$. Calculated, $m / z: 277.1022$. Second eluted a colourless liquid, cis-3-butyltetrahydro-2H-pyran-4-yl 2,2,2-trifluoroacetate $(c-4 a)$. Yield $0.89 \mathrm{~g}(5 \%) . R_{\mathrm{f}}=0.2\left(20 \% \mathrm{Et}_{2} \mathrm{O}\right.$ in petrol). IR spectrum (film), $v, \mathrm{~cm}^{-1}: 775 \mathrm{~m}, 1031 \mathrm{w}, 1121 \mathrm{~m}, 1158 \mathrm{~m}$, 1219 m, 1781 s, 2862 w, 2933 w, 2961 w. ${ }^{1} \mathrm{H}$ NMR spectrum $\left(400 \mathrm{MHz}, \mathrm{CDCl}_{3}\right), \delta$, ppm $(J, \mathrm{~Hz}): 0.88\left(3 \mathrm{H}, \mathrm{t}, J=7, \mathrm{CH}_{3}\right)$; $1.18-1.33\left(6 \mathrm{H}, \mathrm{m}, 3 \mathrm{xCH}_{2}\right)$; $1.84-1.98(3 \mathrm{H}, \mathrm{m}, 3-\mathrm{CH}$ and 5$\left.\mathrm{CH}_{2}\right) ; 3.51\left(1 \mathrm{H}, \mathrm{t}, J=11,2-\mathrm{CH}_{\mathrm{a}}\right) ; 3.63-3.79\left(3 \mathrm{H}, \mathrm{m}, 2-\mathrm{CH}_{\mathrm{b}}\right.$ and $\left.6-\mathrm{CH}_{2}\right) ; 5.32(1 \mathrm{H}, \mathrm{q}, J=3.5,4-\mathrm{CH}) .{ }^{13} \mathrm{C} \mathrm{NMR}$ spectrum $\left(100 \mathrm{MHz}, \mathrm{CDCl}_{3}\right), \delta$, ppm $(J, \mathrm{~Hz})$ : $13.9\left(\mathrm{CH}_{3}\right) ; 22.8(\mathrm{C}-9)$; 26.5 (C-7); 28.7 (C-8); 30.1 (C-5); 39.2 (C-3); 63.0 (C-6); $67.8(\mathrm{C}-2) ; 74.9(\mathrm{C}-4) ; 114.7\left(\mathrm{Q}, J_{\mathrm{C}-\mathrm{F}}=286, \mathrm{CF}_{3}\right) ; 157.1(\mathrm{q}$, $\left.J_{\mathrm{C}-\mathrm{F}}=42, \mathrm{C}=\mathrm{O}\right) .{ }^{19} \mathrm{~F}$ NMR spectrum $\left(376 \mathrm{MHz}, \mathrm{CDCl}_{3}\right), \delta$, ppm: -75.1. Found, $m / z$ : $141.1274\left[\mathrm{M}-\mathrm{OCOCF}_{3}\right]^{+} . \mathrm{C}_{9} \mathrm{H}_{17} \mathrm{O}$. Calculated, $m / z: 141.1273$.

Trans and cis-3-butyltetrahydro-2H-pyran-4-ol (t-4b and $c-4 b)$. Following the above procedure and reaction scale for $\boldsymbol{t} \mathbf{- 4 a}$ and $\boldsymbol{c} \mathbf{- 4 a}$, but after $3 \mathrm{~h}$ at $\mathrm{rt}$, the mixture was neutralised by aq. $\mathrm{NH}_{3}(15 \mathrm{ml})$. The aq. layer was extracted with $\mathrm{Et}_{2} \mathrm{O}(2 \times 30 \mathrm{ml})$, dried $\left(\mathrm{Na}_{2} \mathrm{SO}_{4}\right)$ and evaporated under reduced pressure. The residue, a yellow oil $(\sim 8 \mathrm{~g})$, was distilled at $\sim 15$ mbar and the fraction of bp $100-110^{\circ} \mathrm{C}(4.7$ $\mathrm{g}$, an impure 75:25 mixture of $\boldsymbol{t} \mathbf{- 4 b}$ and $\boldsymbol{c}-\mathbf{4 b}$ ) further purified by flash column chromatography $\left(20-40 \% \mathrm{Et}_{2} \mathrm{OAc}\right.$ in petrol). First eluted a colourless liquid, trans-3butyltetrahydro-2H-pyran-4-ol $\quad(\boldsymbol{t}-\mathbf{4 b}) . \quad$ Yield $1.72 \mathrm{~g}$ $(17 \%) \cdot R_{\mathrm{f}}=0.3$ (30\% EtOAc in petrol). IR spectrum (film), $\mathrm{v}, \mathrm{cm}^{-1}: 626 \mathrm{~s}, 1050 \mathrm{~s}, 1080 \mathrm{~s}, 1150 \mathrm{~s}, 1222 \mathrm{~m}, 1466 \mathrm{~m}, 2856$ s, 2925 s, 2955 s, 3386 br. ${ }^{1} \mathrm{H}$ NMR spectrum $(400 \mathrm{MHz}$, $\left.\mathrm{CDCl}_{3}\right), \delta$, ppm $(J, \mathrm{~Hz}): 0.88\left(3 \mathrm{H}, \mathrm{t}, J=7, \mathrm{CH}_{3}\right) ; 1.03-1.13$ (1 H, m, 7- $\left.\mathrm{CH}_{\mathrm{a}}\right) ; 1.17-1.37\left(4 \mathrm{H}, \mathrm{m}, 8,9-\mathrm{CH}_{2}\right) ; 1.43-1.62(2$ $\mathrm{H}, \mathrm{m}, 3-\mathrm{CH}$ and $\left.5-\mathrm{CH}_{\mathrm{a}}\right) ; 1.63-1.72\left(1 \mathrm{H}, \mathrm{m}, 7-\mathrm{CH}_{\mathrm{b}}\right) ; 1.73-$
$1.92(2 \mathrm{H}, \mathrm{m}, 5-\mathrm{CH}$ and $\mathrm{OH}) ; 3.02\left(1 \mathrm{H}, \mathrm{t}, J=11,2-\mathrm{CH}_{\mathrm{a}}\right)$; $3.35-3.44\left(2 \mathrm{H}, \mathrm{m}, 4-\mathrm{CH}\right.$ and $\left.6-\mathrm{CH}_{\mathrm{a}}\right) ; 3.90-3.98(2 \mathrm{H}, \mathrm{m}, 2-$ $\mathrm{CH}$ and $\left.6-\mathrm{CH}_{\mathrm{b}}\right) .{ }^{13} \mathrm{C} \mathrm{NMR}$ spectrum $\left(100 \mathrm{MHz}, \mathrm{CDCl}_{3}\right), \delta$, ppm: $14.1\left(\mathrm{CH}_{3}\right) ; 23.2$ (C-9); $28.3(\mathrm{C}-7) ; 29.3(\mathrm{C}-8) ; 35.3$ (C-5); 44.6 (C-3); 66.5 (C-6); 70.6 (C-2); 72.1 (C-4). Found, $m / z$ : $141.1272[\mathrm{M}-\mathrm{OH}]^{+} . \mathrm{C}_{9} \mathrm{H}_{17} \mathrm{O}$. Calculated, $m / z$ : 141.1273. Second eluted a colourless liquid, cis-3butyltetrahydro-2H-pyran-4-ol (c-4b). Yield $0.36 \mathrm{~g}(3 \%) . R_{\mathrm{f}}$ $=0.2\left(30 \%\right.$ EtOAc in petrol). IR spectrum (film), $\mathrm{v}, \mathrm{cm}^{-1}: 626$ s, $1080 \mathrm{~s}, 1150 \mathrm{~s}, 1222 \mathrm{~m}, 1466 \mathrm{~m}, 2856 \mathrm{~s}, 2927 \mathrm{~s}, 2955 \mathrm{~s}$, 3389 br. ${ }^{1} \mathrm{H}$ NMR spectrum $\left(400 \mathrm{MHz}, \mathrm{CDCl}_{3}\right), \delta$, ppm $(J$, $\mathrm{Hz}): 0.89\left(3 \mathrm{H}, \mathrm{t}, J=7, \mathrm{CH}_{3}\right) ; 1.19-1.36\left(6 \mathrm{H}, \mathrm{m}, 3 \mathrm{xCH}_{2}\right)$; $1.58(1 \mathrm{H}, \mathrm{br}, \mathrm{OH}) ; 1.63-1.73\left(2 \mathrm{H}, \mathrm{m}, 3-\mathrm{CH}\right.$ and $\left.5-\mathrm{CH}_{\mathrm{a}}\right)$; $1.77-1.86\left(1 \mathrm{H}, \mathrm{m}, 5-\mathrm{CH}_{\mathrm{b}}\right) ; 3.51-3.56\left(2 \mathrm{H}, \mathrm{m}, 2-\mathrm{CH}_{2}\right) ; 3.64$ $\left(1 \mathrm{H}, \mathrm{ddd}, J=11.5, J=4.5, J=3.5,6-\mathrm{CH}_{\mathrm{a}}\right) ; 3.78(1 \mathrm{H}, \mathrm{td}, J$ $\left.=11, J=3,6-\mathrm{CH}_{\mathrm{b}}\right) ; 4.00(1 \mathrm{H}, \mathrm{dt}, J=5.5, J=3.0,4-\mathrm{CH})$. ${ }^{13} \mathrm{C}$ NMR spectrum $\left(100 \mathrm{MHz}, \mathrm{CDCl}_{3}\right), \delta$, ppm: $14.1\left(\mathrm{CH}_{3}\right)$; 23.1 (C-9); 26.6 (C-7); 29.1 (C-8); 33.5 (C-5); 40.9 (C-3); $63.2(\mathrm{C}-6)$; 66.7 (C-4); $67.6(\mathrm{C}-2)$. Found, $m / z$ : 141.1272 $[\mathrm{M}-\mathrm{OH}]^{+} . \mathrm{C}_{9} \mathrm{H}_{17} \mathrm{O}$. Calculated, $m / z: 141.1273$.

Trans-3-butyltetrahydro-2H-pyran-4-ol (t-4b). Trans3-butyltetrahydro-2H-pyran-4-yl 2,2,2-trifluoroacetate ( $t$ 4a) $(250 \mathrm{mg}, 0.98 \mathrm{mmol})$ was stirred with $\mathrm{K}_{2} \mathrm{CO}_{3}(270 \mathrm{mg}$, $1.96 \mathrm{mmol})$ in $\mathrm{MeOH}(1 \mathrm{ml})$ at rt. After $3 \mathrm{~h}$, the mixture was quenched with $10 \% \mathrm{HCl}(1 \mathrm{ml})$ and extracted with EtOAc $(2 \times 5 \mathrm{ml})$. The combined organic layers were washed with brine $(2 \mathrm{ml})$ and dried $\left(\mathrm{Na}_{2} \mathrm{SO}_{4}\right)$. Evaporation under reduced pressured followed by chromatography (30\% EtOAc in petrol) gave a colourless liquid, trans-3-butyltetrahydro- $2 \mathrm{H}$ pyran-4-ol (t-4b). Yield $138 \mathrm{mg}(89 \%)$. Data as above.

Cis-3-butyltetrahydro-2H-pyran-4-ol (c-4b). Cis-3butyltetrahydro-2H-pyran-4-yl 2,2,2-trifluoroacetate (c-4a) (150 mg, $0.59 \mathrm{mmol}$ ) was stirred with $\mathrm{K}_{2} \mathrm{CO}_{3}$ (163 mg, 1.18 mmol) in $\mathrm{MeOH}(1 \mathrm{ml})$ at $\mathrm{rt}$. After $3 \mathrm{~h}$, the mixture was quenched with $10 \% \mathrm{HCl}(1 \mathrm{ml})$ and extracted with EtOAc $(2 \times 5 \mathrm{ml})$. The combined organic layers were washed with brine $(2 \mathrm{ml})$ and dried $\left(\mathrm{Na}_{2} \mathrm{SO}_{4}\right)$. Evaporation under reduced pressured followed by chromatography $(30 \%$ EtOAc in petrol) gave a colourless liquid, cis-3-butyltetrahydro- $2 \mathrm{H}$ pyran-4-ol (c-4b). Yield $87 \mathrm{mg}$ (93\%). Data as above.

The Supplementary information file containing tabular comparisons of ${ }^{13} \mathrm{C}$ NMR data of $\boldsymbol{t}-\mathbf{4 a}, \boldsymbol{c}-\mathbf{4 a}, \boldsymbol{t}-\mathbf{4 b}$ and $\boldsymbol{c}-\mathbf{4 b}$ with that from Talipov's work and NMR spectra of $\boldsymbol{t}-\mathbf{4 a}, \boldsymbol{c}$ $\mathbf{4 a}, \boldsymbol{t}-\mathbf{4 b}$ and $\boldsymbol{c}-\mathbf{4 b}$ is available from the journal website at http://hgs.osi.lv.

The authors thank the Higher Committee for Education Development in Iraq for studentship support (to H.A.A.A.), the EPSRC and Roche for a CASE award (to M.A.H.S.), and Prof. Talipov (Bashkir State University) for useful correspondence.

${ }^{\dagger}$ Email from Prof. R. F. Talipov, 13th February 2008.

\section{References}

1. Olier, C.; Kaafarani, M.; Gastaldi, S.; Bertrand, M. P. Tetrahedron 2010, 66, 413.

2. (a) Arundale, E.; Mikeska, L. A. Chem. Rev. 1952, 51, 505. (b) Adams, D. R.; Bhatnagar, S. P. Synthesis 1977, 661. (c) Pastor, I. M.; Yus, M. Curr. Org. Chem. 2007, 11,925 . 
3. (a) Stapp, P. R. J. Org. Chem. 1969, 34, 479. (b) Onopchenko, A.; Seekircher, R. J. Chem. Eng. Data 1970, 15,164 .

4. (a) Solov'eva, N. P.; Smol'yaninova, E. K.; Belov, V. N. Zh. Obshch. Khim. 1957, 27, 3015. (b) Heslinga, L.; VanGorkom, M. Recl. Trav. Chim. Pays-Bas 1966, 85, 293. (c) Solov'eva, N. P.; Tsirkel, T. M.; Terekhina, I. A.; Rudol'fi, T. A.; Voitkevich, S. A. Chem. Heterocycl. Compd. 1971, 7, 530. [Khim. Geterotsikl. Soedin. 1971, 1447.] (d) Stapp, P. R. J. Org. Chem. 1970, $35,2419$.

5. (a) Talipov, R. F.; Starikov, A. S.; Gorina, I. A. Akmanova, N. A.; Safarov, M. G. Russ. J. Org. Chem. 1993, 29, 844. [Zh. Org. Khim. 1993, 29, 1024.] (b) Igdavletova, M. Z.; Starikov, A. S.; Talipov, R. F.; Akmanova, N. A.; Safarov, I. M. Pet. Chem. 1993, 29, 420. [Neftekhimiya 1993, 33, 436.] (c) Talipov, R. F.; Starikov, A. S.; Gorin, A. V.; Safarov, M. G. Russ. J. Org. Chem. 1993, 29, 624. [Zh. Org. Khim. 1993, 29, 748.]

6. Talipov, R. F.; Starikov, A. S.; Akmanova, N. A.; Safarov, M. G. RU 2043348 C1 1995; Chem, Abstr. 1996:273611.

7. Talipov, R. F.; Safarov, M. G. Bashk. Khim. Zh. 1996, 3, 119.

8. (a) Talipov, R. F.; Safarov, I. M.; Talipova, G. R.; Safarov, M. G. React. Kinet. Catal. Lett. 1997, 61, 63. (b) Talipov, R. F.; Yusupov, Z. A.; Safarov, I. M.; Talipova, R. G.; Muslukhov, R. R. Izv. Vyss. Uchebn. Zaved., Khim. Khim. Tekhnol. 1998, 41, 131. (c) Syrlybaeva, R. R.; Vakulin, I. V.; Talipov, R. F. React. Kinet., Mech. Catal. 2013, 109, 301. (d) Vakulin, I. V.; Syrlybaeva, R. R.; Talipov, R. F.; Talipova, G. R.; Faizullina, R. R.; Allagulova, A. V. In Key Technologies in Polymer Chemistry, Morozkin, N.D.; Zakharov, V. P.; Zaikov, G. E., Eds.; CRC Press: Boca Raton, 2015, p. 75.

9. Talipov, R. F.; Muslukhov, R. R.; Safarov, I. M.; Yamantaev, F. A.; Safarov, M. G. Chem. Heterocycl. Compd. 1995, 31, 530. [Khim. Geterotsikl. Soedin. 1995, 605.]

10. (a) Gianturco, M. A.; Friedel, P. Can. J. Chem. 1966, 44, 1083. (b) 3-pentyl-2,5-dihydrofuran, prepared by us (Hodgson, D. M.; Stent, M. A. H.; Wilson, F. X. Synthesis 2002, 1445.), displayed an olefinic proton [(400 $\left.\mathrm{MHz}, \mathrm{CDCl}_{3}\right), \delta$, ppm: 5.43-5.48 (1 H, m, C=CH)] consistent with ref 10 (a).

11. Forzato, C.; Nitti, P.; Pitacco, G.; Valentin, E. Tetrahedron: Asymmetry, 1997, 8, 1811.

12. Bentley, J.; Nilsson, P. A.; Parsons, A. F. J. Chem. Soc. Perkin Trans. 1 2002, 1461.

13. Clive, D. L. J.; Yang, W.; MacDonald, A. C.; Wang, Z.; Cantin, M. J. Org. Chem. 2001, 66, 1966.

14. Nikolic, N. A.; Gonda, E.; Longford, C. P. D.; Lane, N. T.; Thompson, D. W. J. Org. Chem. 1989, 54, 2748.

15. A similar result was observed at $60^{\circ} \mathrm{C}$. 